\title{
Aging of Silver Iodide-Sodium Iodide Generator Effluent in Moist and Dry Air
}

\author{
Donald N. Blair and Briant L. Davis \\ Institute of Atmospheric Sciences, South Dakota School of Mines and Technology, Rapid City
}

(Manuscript received 2 January 1969, in revised form 14 April 1969)

\begin{abstract}
The effect of storage of AgI-NaI aerosol particles in moist and dry air upon the efficiency of nucleation of such particles has been investigated. Samples were obtained from a standard solution generator and tested in an optical cold stage at $-15 \mathrm{C}$ under a controlled atmosphere. The "wet" and "dry" samples were paired and studied together in the optical cold chamber to eliminate several environmental variables. In 16 out of 21 runs the aerosol aged under dry conditions showed a higher nucleation efficiency, there also being a possible weak positive correlation of the ratio of efficiencies of dry to wet samples with time. Differences between these results and those of other workers can best be explained by differences in aging treatment.
\end{abstract}

\section{Introduction}

Recently a number of workers have suggested that the presence of a water envelope around nucleant particles may affect the efficiency of nucleation of such particles. Mason (1968), Gokhale (1965) and Hoffer (1961) have observed a deterioration of the nucleation ability for a number of materials, including silver iodide, when immersed in pure water. However, no attempt was made to insure true "dryness" of the untreated nuclei in their studies. St. Louis and Steele (1968) have shown experimentally that the presence of water vapor enhances the nucleation efficiency for $\mathrm{AgI}-\mathrm{NaI}$ aerosols produced from acetone solution generators. Here drying was accomplished by storing the aerosol in nitrogen gas with storage times of up to 100 min. Birstein (1952) has likewise shown an increase in the efficiency of nucleation with exposure to various humidities of silver iodide nuclei prepared by vaporization of fused AgI crystals from a hot nichrome coil. This effect is relative to those obtained from aerosols stored in dry nitrogen for up to several hours. Hoffer and Ogne (1965) observed a decrease in nucleation effectiveness for pure sieved reagent-grade AgI after humidification treatment which resulted in water envelopes around each particle. After drying from 5-15 min, these particles were $20 \%$ less effective at $-4 \mathrm{C}$ than particles not undergoing humidification. Phloroglucinol showed the opposite effect, however. Inasmuch as no hygroscopic impurity was present, the AgI particles were subjected to $200 \%$ relative humidity to develop the water envelopes.

There is a fundamental difference in the methods used by Mason, Gokhale, and Hoffer from those of St. Louis and Steele, and Birstein. In each case liquid surrounds the nuclei, but in the former the nuclei are physically introduced into the pure water and in the latter any moisture collecting upon the nuclei takes place by virtue of the hygroscopic ability of the particles. Birstein irradiated his samples with ultraviolet light and observed a lessening of the UV-related deterioration as the samples were exposed to higher relative humidities. The opposite effect was noted by St. Louis and Steele in those experiments involving ultraviolet irradiation.

Smith et al. (1958), in studying the effect of sunlight on activity of AgI nuclei released from aircraft, indicated that other mechanisms of decay may have been responsible for the variability of their observations. In this study, we eliminate the irradiation variable by storing the sample in darkness and are concerned only with the effects of hygroscopically absorbed moisture. The particles are thus aged as a result of immersion in their hygroscopically derived solution envelopes.

\section{Experimental procedure}

The effluent particles are produced in our laboratory by the burning of AgI-NaI-acetone solutions (3\% AgI by weight; $0.5: 1$ mole ratio $\mathrm{AgI}: \mathrm{NaI}$ ) in which the solution was forced through a hypodermic needle into a propane flame. In these respects, the generator is similar to that used by St. Louis and Steele. The flow rate of the solution was kept sensibly constant at about $2.6 \mathrm{~cm}^{3} \mathrm{~min}^{-1}$.

The experiments were completed in two series, hereafter called "wet" and "dry" runs. All aerosol particles were precipitated in sealed beakers on small one-fourth inch glass slides while stored in darkness. After $15 \mathrm{~min}$ the dry particles were placed in a dessicator (dry runs), while others were exposed to moist air of $>90 \%$ relative humidity at room temperature (wet runs) for lengths of time varying from $30-220 \mathrm{~min}$. The time required to transfer the samples from the precipitation beaker to 


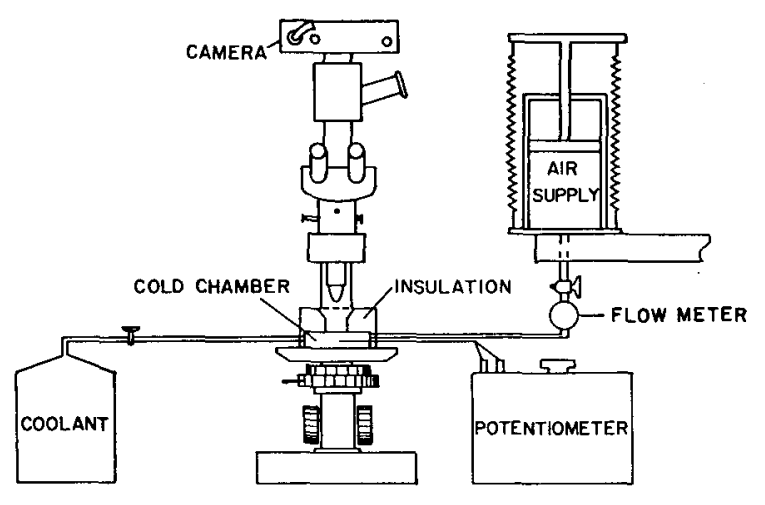

A.

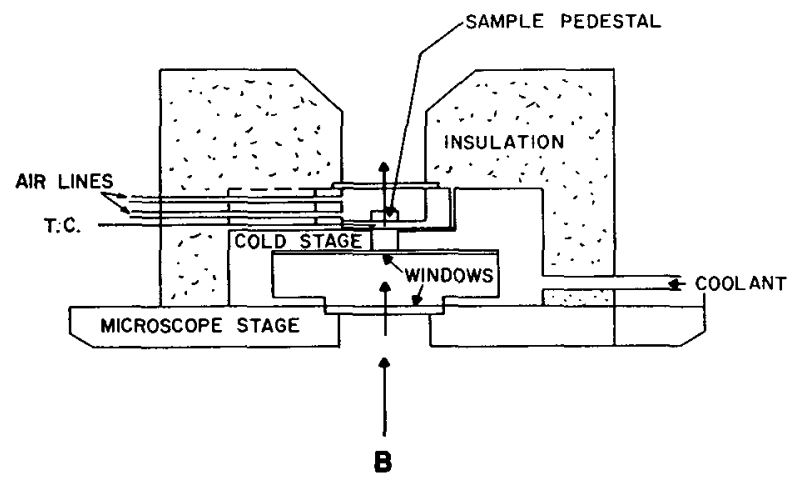

FIG. 1. Schematic drawing of optical cold stage assembly (A); and details of cold chamber showing air lines, thermocouple (T.C.), light windows, and coolant space (B). The microscope stage is 6 inches in diameter.

their respective aging environments was $<30 \mathrm{sec}$. One wet and one dry sample were paired and placed into a sample well of a commercial optical cold chamber and sealed with a coverglass and vacuum grease.

The cold chamber well was kept sealed and dry (with nitrogen vapor) until it had been cooled slightly below $-20 \mathrm{C}$ and had then warmed to this value. Air of dew point $-1 \pm 1 \mathrm{C}$ was then passed over the pair of samples for 1 min. Air of this moisture level will not freeze up the small air conduits but gives sufficient moisture to insure that the growth rate of crystals in this period of time was significant. The sample temperature was found to be $-15 \mathrm{C}$ in the presence of the $80 \mathrm{~cm}^{3} \mathrm{~min}^{-1}$ air flow determined by blank runs with thermocouples placed directly on the glass pedestal surface. In the presence of water, the saturation ratio over the sample within the chamber is solely a function of the sample temperature and was 1.16 at $-1.5 \mathrm{C}$.

In effect we are measuring the nucleation efficiency of the aerosols as a function of a "long-term" aging and are assuming that the short period during which the samples are in the cold chamber under nitrogen vapor does not alter the physical or chemical effects brought about by the aging process. Under these conditions the moisture envelope formed over the wet sample particle may be greatly reduced or eliminated entirely, which for the purposes of this investigation is desirable. Thus, the aerosol samples are in all respects similar at the start of the nucleation period, except for the long-term aging process. None of the particles showed visible water during any stage in the chamber test.

Fig. 1A shows the experimental arrangement with microscope, cold stage, air supply, and potentiometer with attached thermocouple. Fig. $1 \mathrm{~B}$ presents a detailed view of the cold chamber.

\section{Results and discussion}

Fig. 2A, representing a typical run, is a photograph of ice crystals grown from both wet and dry aerosol particles. The larger of the two surfaces (separated by the sharp contact between the two slide edges) contained the dry aerosol and is typical of the uniformity of crystal density obtained with this method. It should be recalled that both surfaces shown have the same initial aerosol densities inasmuch as they were placed side by side in the same precipitation beaker with the same aerosol injection.

Figs. 3-5 present the results of all runs at various temperatures, plotted as measured crystal density $D_{w}$ and $D_{d}, D_{d}-D_{w}$, and $D_{d} / D_{w}$ vs time, respectively. Fig. 3 presents the initial data but Figs. 4 and 5 more adequately illustrate the trends resulting from aging. In only 5 of the 21 pairs did the aerosol aged under moist air conditions (wet sample) produce more nuclei than did the dry samples. In spite of considerable scatter the greater nucleation efficiency of the dry sample is obvious. Due to the excellent uniformity in crystal densities obtained with this method, as well as the fact that little crystal clustering was observed, we estimate the uncertainty in crystal density measurement to be $<15 \%$. The fact that the samples were studied in pairs is vital in this regard as well as in the elimination of other possible variables such as chamber temperature, input air flow rate, etc., that would have to be considered in taking wet and dry runs separately. A remaining uncertainty is the difference in cleanliness of the glass plates used but sufficient runs were made to eliminate any systematic biasing of the selection. Moreover, the position of the slides within the chamber were alternately switched to avoid any inherent differences in air flow from one position to another.

The effect of contact of the aerosol particles on the glass slides appears to be a general lowering of the efficiency of the aerosol, as might be expected from surface area considerations only. We observe efficiencies on the order of only $10^{12}$ nuclei $\mathrm{gm}^{-1} \mathrm{AgI}$ at $-15 \mathrm{C}$ for the generator when analyzed in this manner. This fact should have no effect on the relative effciencies of the aged particles which is our concern in this investigation.

The only other study involving AgI-NaI aerosols produced from a generator was that of St. Louis and Steele. Their results on such aerosols conditioned for up 


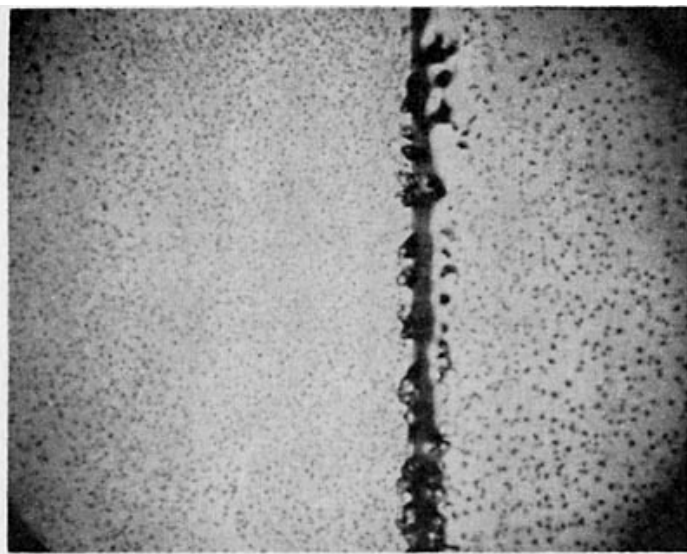

(A)

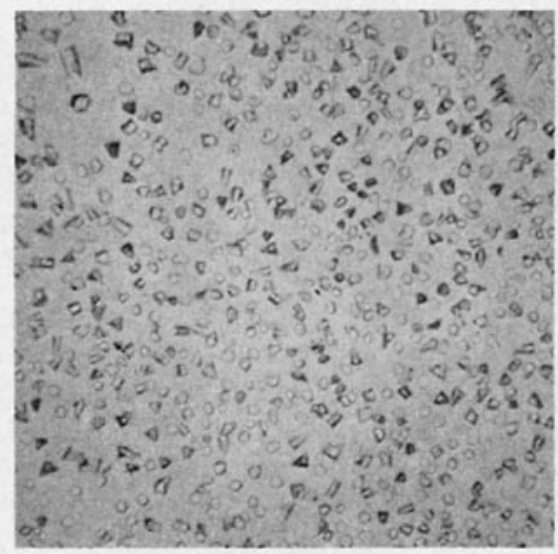

(B)

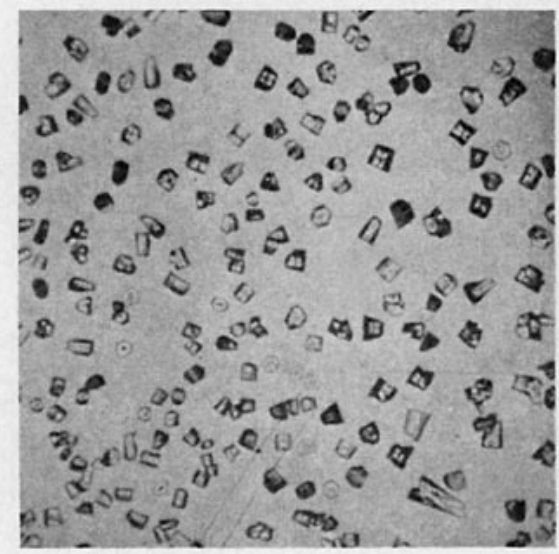

(C)

FIG. 2. Paired sample surfaces at $25 \times$ in microscope after 1 min exposure to air saturated with respect to water at $-15 \mathrm{C}$; aerosol aged under dry conditions on left side of vertical contact boundary (A): (B) and (C), higher power magnification $(\times 75)$ of dry and wet surface in (A), respectively.

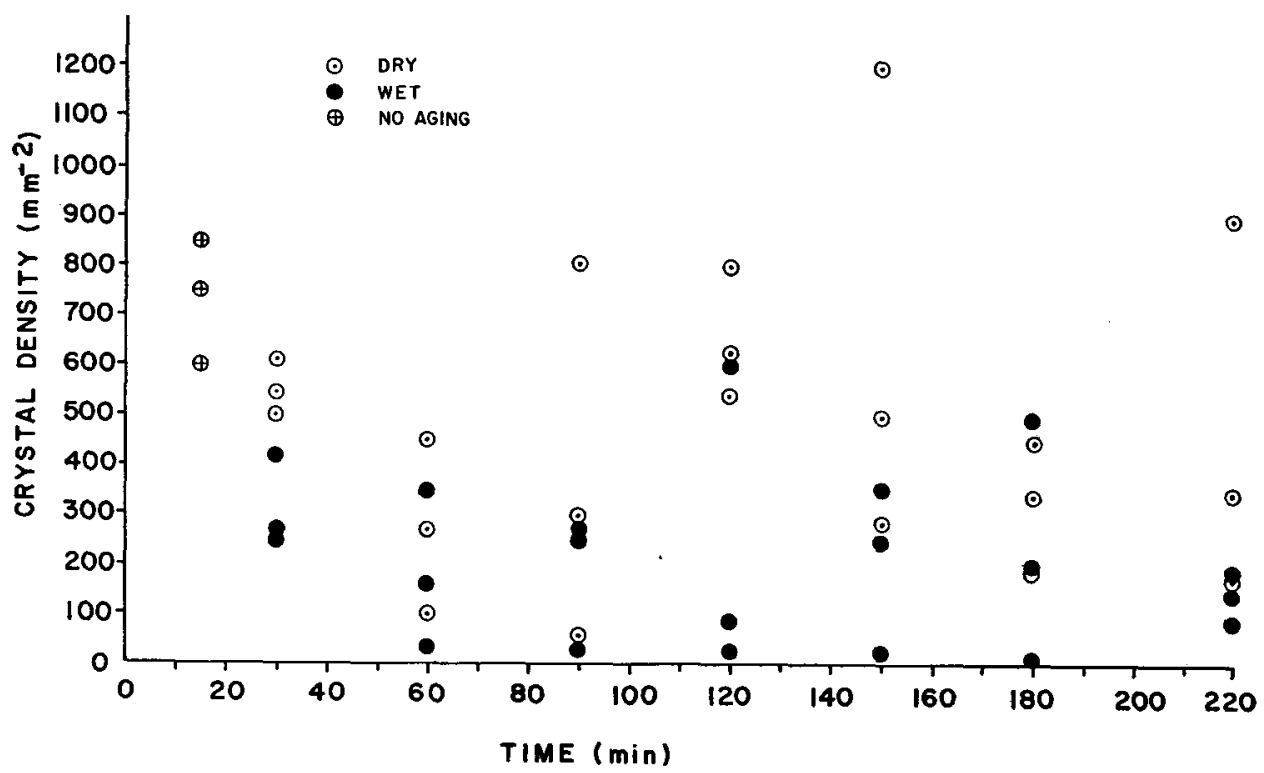

FIG. 3. Ice crystal densities obtained from nucleation at $-15 \mathrm{C}$ of wet and dry paired aerosol surfaces plotted vs time of aging. 


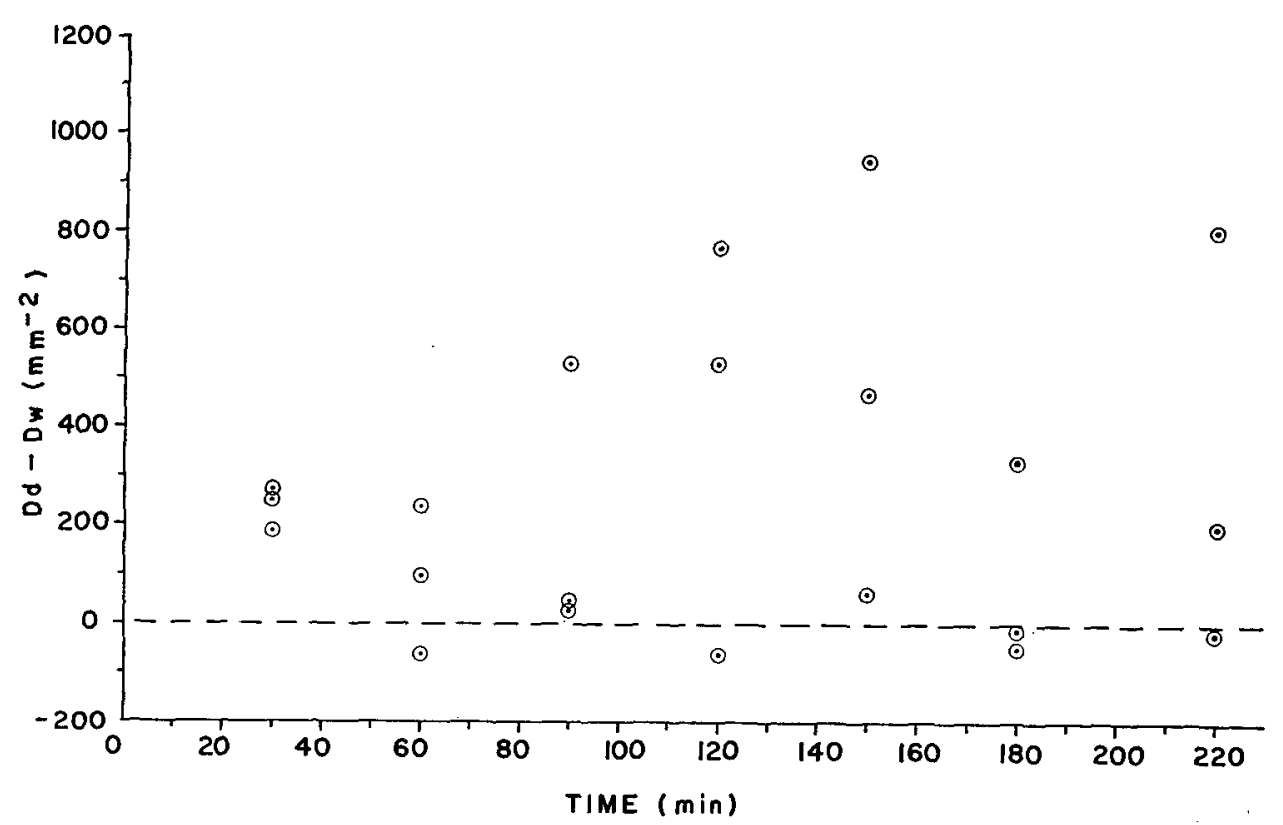

FIG. 4. Differences in observed ice crystal densities from paired wet and dry samples plotted vs time of aging; nucleation temperature, $-15 \mathrm{C}$.

to $100 \mathrm{~min}$ in the presence of water vapor pressures to near $24 \mathrm{~mm} \mathrm{Hg}$, and compared with control samples held at zero vapor pressure, showed an increase in the relative effectiveness of the wet over dry aerosols. After the first $20 \mathrm{~min}$, however, no further change in this difference was noted.

In the present study we tentatively conclude that the presence of water vapor has a suppressive effect on nucleation ability. The scatter in the data leave some doubt as to the variation of this effect with time, inasmuch as correlation coefficients for the wet and dry data of Fig. 3 are -0.11 and 0.03 , respectively. In Fig. 5, if one considers the individual ratios of pairs of wet and dry samples (as paired experimentally), this parameter increases to 0.23 . Since runs were repeated three times for each temperature a reduction in scatter about a real trend with time might be expected by averaging the three values before making a correlation test. When this is done the coefficient becomes 0.70 . The dashed line of Fig. 5 was determined from the unaveraged data by least squares.

The enhanced activity of wet over dry samples observed by St. Louis and Steele may well be the result of the presence of a considerable water envelope surrounding the aerosol particles when introduced into the cold chamber. The increase in nucleation effectiveness with increase in water vapor pressure present during aging, also observed by St. Louis and Steele, supports this suggestion and may even imply that at a given temperature a critical size water envelope must be developed prior to freezing. Such an envelope was also undoubtedly present during the aging period of the wet samples

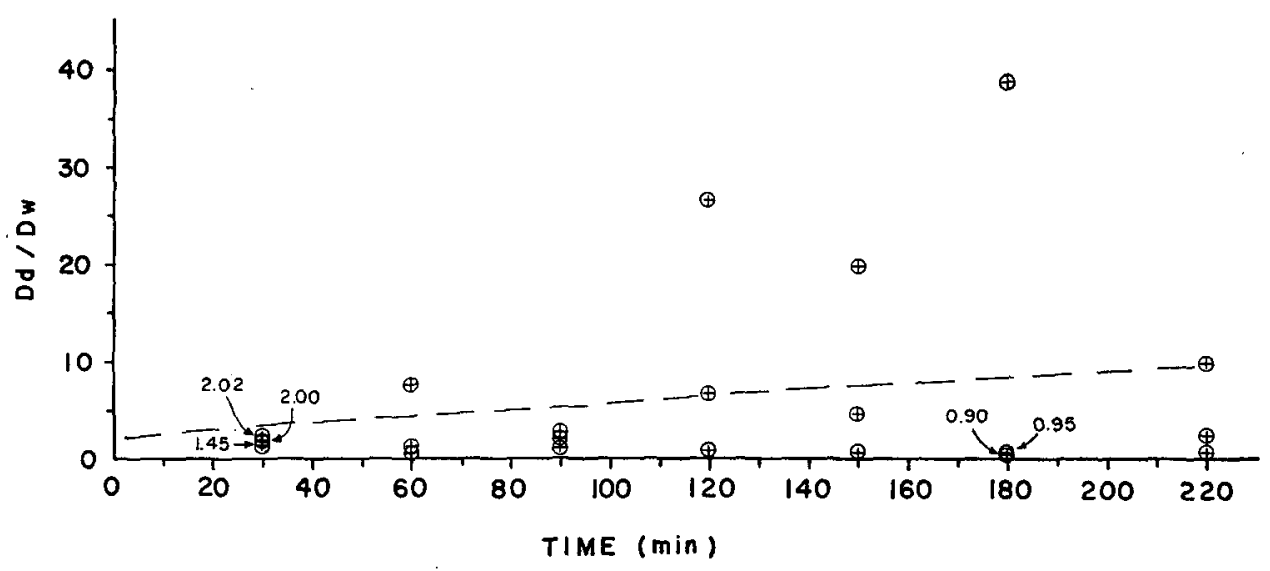

FIG. 5. Ratios of crystal densities for paired samples of wet and dry aerosol plotted vs time of aging; nucleation temperature, $-15 \mathrm{C}$. 
studied here, but these were greatly reduced or eliminated during momentary storage in the chamber prior to the nucleation test. Thus, with no significant difference in initial water content present at the start of the nucleation test, the nucleation efficiency would be more a function of the physical or chemical processes that had modified the nucleation sites during aging. From a strain energy standpoint (to be discussed in a future paper) we believe that etching of the AgI particle in the presence of water and $\mathrm{NaI}$ should destroy in part the nucleating ability of the particle. Work on these problems is continuing in this laboratory.

Acknowledgments. This project was financially supported by the Office of Atmospheric Water Resources, Bureau of Reclamation, U. S. Dept. of the Interior, under Contract 14-06-D-5979.

\section{REFERENCES}

Birstein, S. J., 1952: The effect of relative humidity on the nucleating properties of photolyzed silver iodide. Bull. Amer. Meteor. Soc., 33, 431-434.

Gokhale, N. R., 1965: Comparison of ice nucleating efficiencies of chemical aerosols in a supercooled cloud and in bulk water. Proc. Intern. Conf. Cloud Physics, Tokyo, 176-180.

Hoffer, T. E., 1961 : A laboratory investigation of droplet freezing. J. Meteor., 18, 766-778.

- - and M. L. Ogne, 1965: A difference in the behavior of phloroglucinol and silver iodide nuclei. $J$. Geophys. Res., 70, 3857-3860.

Mason, B. J., 1968: Initiation of the ice phase in the atmosphere. Proc. Intern. Conf. Cloud Physics, Toronto, 162-173.

St. Louis, P., and R. Steele, 1968: Certain environmental effects on silver iodide ice nuclei. Proc. Intern. Conf. Cloud Physics, Toronto, 178-182.

Smith, E. J., K. J. Herrernan and W. J. Thompson, 1958: The decay of the ice-nucleating properties of silver iodide released from an aircraft. Quart. J. Roy. Meteor. Soc., 84, 162-165. 